Pacific Journal of Mathematics

PRIMITIVE ALGEBRAS WITH INVOLUTION

Wallace Smith Martingale, II 


\title{
PRIMITIVE ALGEBRAS WITH INVOLUTION
}

\author{
Wallace S. Martindale, 3RD
}

A well known theorem of Kaplansky ([1], p. 226, Theorem 1) states that every primitive algebra satisfying a polynomial identity is finite dimensional over its center. Related to this result is the following conjecture due to Herstein: if $A$ is a primitive algebra with involution whose symmetric elements satisfy a polynomial identity, then $A$ is finite dimensional over its center. Our main object in the present paper is to verify this conjecture in the special case where $A$ is assumed to be algebraic. In the course of our proof we develop some results, which may be of independent interest, concerning the existence of nontrivial symmetric idempotents in primitive algebras with involution.

1. Some preliminary remarks. In the present section we mention a few definitions and observations which we shall need in the remainder of this paper.

By the term algebra over $\Phi$ we shall mean an associative algebra (possibly infinite dimensional) over a field $\Phi$. A primitive algebra over $\Phi$ is one which is isomorphic to a dense ring of linear transformations of a (left) vector space $V$ over a division algebra $\Delta$ containing $\Phi$ (see [1], p. 32). The rank of an element $a$ of a primitive algebra is the dimension of $V a$ over $\Delta$. We state without proof the following three remarks.

Remark 1 . Let $A$ be a primitive algebra with identity 1 containing a set of nonzero orthogonal idempotents $e_{1}, e_{2}, \cdots, e_{m}$ such that

(a) $e_{1}+e_{2}+\cdots+e_{m}=1$

(b) $\operatorname{rank} e_{i}=r_{i}<\infty, i=1,2, \cdots, m$.

Then the dimension of $V$ over $\Delta$ is $\sum_{i=1}^{m} r_{i}<\infty$.

Remark 2. Let $A$ be a primitive algebra with center $Z$. If $z a=0$ for some $z \neq 0 \in Z$ and some $a \in A$, then $a=0$.

REMARK 3. Let $A$ be a primitive algebra. If $a$ and $b$ are nonzero elements of $A$, then $a A b \neq 0$. More generally, if $a_{1}, a_{2}, \cdots, a_{n}$ are nonzero elements of $A$, where $n$ is any natural number, then

$$
a_{1} A a_{2} A \cdots a_{n-1} A a_{n} \neq 0 \text {. }
$$

An I-algebra is an algebra in which every non-nil left ideal contains a nonzero idempotent. An algebra over $\Phi$ is algebraic in case every

Received September 23, 1960. 
element satisfies a non-trivial polynomial equation $f(t)=0$, where $f(t)=$ $\sum \alpha_{i} t^{i}, \alpha_{i} \in \Phi$. One can show that every algebraic algebra is an $I$-algebra. In the proof of this fact (see [1], p. 210, Proposition 1), however, the following sharper result is obtained.

REMARK 4. Let $a$ be a non-nilpotent element of an algebraic algebra. Then the subalgebra $[[a]]$ generated by $a$ contains a nonzero idempotent.

An involution* of an algebra $A$ over $\Phi$ is an anti-automorphism of $A$ of period 2, that is,

$$
\begin{aligned}
& (a+b)^{*}=a^{*}+b^{*} \\
& (\alpha a)^{*}=\alpha a^{*} \\
& (a b)^{*}=b^{*} a^{*} \\
& a^{* *}=a
\end{aligned}
$$

for all $a, b \in A, \alpha \in \Phi$. It is to be understood that in the rest of this paper the characteristic of $\Phi$ is assumed to be unequal to 2. An element $a$ is symmetric if $a^{*}=a ; a$ is skew if $a^{*}=-a .{ }^{*}$ is an involution of the first kind in case every central element is symmetric. ${ }^{*}$ is an involution of the second kind in case there exists a nonzero central element which is skew. Every involution is of one of these two kinds.

2. $S_{n}$-algebras. The notion of an algebra satisfying a polynomial identity can be generalized according to the following

Definition. A subspace $R$ of an algebra $A$ over $\Phi$ satisfies a polynomial identity in case there exists a nonzero element $f\left(t_{1}, t_{2}, \cdots, t_{n}\right)$, of the free algebra over $\Phi$ freely generated by the $t_{i}$ such that

$$
f\left(x_{1}, x_{2}, \cdots, x_{n}\right)=0
$$

for all $x_{i} \in R . \quad R$ will be called a $P I$-subspace of degree $d$ if the degree $d$ of $f\left(t_{1}, t_{2}, \cdots, t_{n}\right)$ is minimal.

The element $f\left(t_{1}, t_{2}, \cdots, t_{n}\right)$ is multilinear of degree $n$ if and only if it is of the form

$$
\sum_{\sigma} \alpha(\sigma) t_{\sigma_{1}} t_{\sigma_{2}} \cdots t_{\sigma_{n}}, \alpha(\sigma) \in \Phi, \text { some } \alpha(\sigma) \neq 0,
$$

where $\sigma$ ranges over all the permutations of $(1,2, \cdots, n)$.

Lemma 1. Let $R$ be a PI-subspace of degree $n$ of an algebra $A$. Then $R$ satisfies a multilinear polynomial identity of degree $n$.

This lemma is a slight generalization of [1], p. 225, Proposition 1. 
The same proof carries over directly and we therefore omit it.

Our main purpose in this paper is to study algebras of the following type.

Definition. Let $A$ be an algebra with an involution ${ }^{*}$ over $\Phi$. Suppose that the set $S$ of symmetric elements is a $P I$-subspace of degree $\leqq n$. Then $A$ will be called an $S_{n}$-algebra. In case ${ }^{*}$ is of the first (second) kind, we shall refer to $A$ as an $S_{n}$-algebra of the first (second) kind.

It is surprisingly easy to analyze $S_{n}$-algebras of the second kind, as indicated by

THEOREM 1. Let $A$ be a primitive $S_{n}$-algebra of the second kind. Then $A$ is finite dimensional over its center.

Proof. ${ }^{1}$ According to Lemma $1 S$ satisfies a multilinear polynomial identity of degree $n: f\left(t_{1}, t_{2}, \cdots, t_{n}\right)=0$. Let $z$ be a nonzero central element of $A$ which is skew. If $k$ is skew, then

$$
(z k)^{*}=k^{*} z^{*}=(-k)(-z)=k z=z k,
$$

and hence $z k$ is symmetric. Therefore we have

$$
0=f\left(z k_{1}, s_{2}, s_{3}, \cdots, s_{n}\right)=z f\left(k_{1}, s_{2}, s_{3}, \cdots, s_{n}\right)
$$

for all $k_{1} \in K, s_{i} \in S$, where $K$ is the set of skew elements. By Remark 2 $f\left(k_{1}, s_{2}, s_{3}, \cdots, s_{n}\right)=0$. It follows that $f\left(x_{1}, s_{2}, s_{3}, \cdots, s_{n}\right)=0$ for all $x_{1} \in A$, $s_{i} \in S$, since every $x \in A$ can be written $x=s+k, s \in S, k \in K$. Continuing in this fashion we finally have $f\left(x_{1}, x_{2}, \cdots, x_{n}\right)=0$ for all $x_{i} \in A$. The conclusion then follows from the previously mentioned theorem of Kaplansky ([1], p. 226, Theorem 1).

3. Some basic theorems. The assumption that the symmetric elements of an $S_{n}$-algebra satisfy a polynomial identity is used chiefly to prove

THEOREM 2. Let $A$ be a primitive $S_{n}$-algebra over $\Phi$. Then there exist at most $n$ orthogonal non-nilpotent symmetric elements.

Proof. Suppose $s_{1}, s_{2}, \cdots, s_{n+1}$ are $n+1$ orthogonal non-nilpotent symmetric elements. Using Remark 3 and the fact that the $s_{i}$ are nonnilpotent we may choose elements $x_{1}, x_{2}, \cdots, x_{n} \in A$ so that

$$
s_{1}^{2} x_{1} s_{2}^{2} x_{2} \cdots s_{n}^{2} x_{n} s_{n+1} \neq 0 .
$$

${ }^{1}$ A similar proof was communicated orally to the author by I. N. Herstein. 
Now set $u_{i}=s_{i} x_{i} s_{i+1}+s_{i+1} x_{i}^{*} s_{i}, i=1,2, \cdots, n$. By Lemma $1 S$ satisfies a multilinear identity of degree $n$ :

$$
f\left(t_{1}, t_{2}, \cdots, t_{n}\right)=t_{1} t_{2} \cdots t_{n}+\sum_{\sigma \neq I} \alpha(\sigma) t_{\sigma_{1}} t_{\sigma_{2}} \cdots t_{\sigma_{n}},
$$

where $\sigma$ ranges over all the permutations of $(1,2, \cdots, n)$ except the identity permutation $I . \quad f\left(u_{1}, u_{2}, \cdots, u_{n}\right)=0$ since the $u_{i}$ are symmetric. To analyze the right hand side of (1) we first note that if $u_{i} u_{j} u_{k} \neq 0$, $i, j, k$ distinct, then either $j=i+1$ and $k=i+2$, or $j=i-1$ and $k=i-2$, because of the orthogonality of the $s_{i}$. It follows that

$$
f\left(u_{1}, u_{2}, \cdots, u_{n}\right)=u_{1} u_{2} \cdots u_{n}+\alpha u_{n} u_{n-1} \cdots u_{1}
$$

for some $\alpha \in \Phi$. Hence

$$
0=s_{1} x_{1} s_{2}^{2} x_{2} s_{3}^{2} x_{3} \cdots s_{n}^{2} x_{n} s_{n+1}+\alpha s_{n+1} x_{n}^{*} s_{n}^{2} x_{n-1}^{*} \cdots s_{2}^{2} x_{1}^{*} s_{1} .
$$

Multiplying (2) through on the left by $s_{1}$, we have $0=s_{1}^{2} x_{1} s_{2}^{2} x_{2} \cdots s_{n}^{2} x_{n} s_{n+1}$, a contradiction.

An idempotent $e$ of an algebra $A$ is called non-trivial in case $e \neq 1$ (if $A$ has an identity) and $e \neq 0$.

THEOREM 3. Let $A$ be a primitive I-algebra with an involution*. Then:

(a) If there exists an $x \neq 0 \in A$ such that $x x^{*}=0$, then either $A$ contains a non-trivial symmetric idempotent or $A$ is isomorphic to the total matrix ring $\Delta_{2}$, where $\Delta$ is a division algebra. In the latter case $E_{11}^{*}=E_{22}$, where the $E_{i j}$ are the nit matrices, $i, j=1,2$.

(b) If $x x^{*} \neq 0$ for all $x \neq 0 \in A$, then either $A$ is a division algebra or $A$ contains a non-nilpotent symmetric element which has no inverse in $A$. If $x x^{*} \neq 0$ for all $x \neq 0 \in A$ and $A$ is algebraic over $\Phi$, then either $A$ is a division algebra or $A$ contains a non-trivial symmetric idempotent.

Proof. Suppose first that there exists an $x \neq 0 \in A$ such that $x x^{*}=0$. We can choose an $a \in A$ such that $e=a x$ is a nonzero idempotent, because $A$ is an $I$-algebra. Since $x x^{*}=0, e \neq 1$. From the equations $e e^{*}=(a x)(a x)^{*}=a x x^{*} a^{*}=0$ it is easy to check that $e+e^{*}-e^{*} e$ is a non zero symmetric idempotent. We may thus assume that $1 \in A$ and $e+e^{*}-e^{*} e=1$. $e A e$ is a primitive $I$-algebra ([1], p. 48, Proposition 1, and p. 211, Proposition 2). If $e A e$ is not a division algebra, then it contains an idempotent $f=e b e, f \neq 0, f \neq e$. Since $f f^{*}=e b e e^{*} b^{*} e^{*}=0$, $f+f^{*}-f^{*} f$ is a nonzero symmetric idempotent. It is unequal to 1 since otherwise $e=e\left(f+f^{*}-f^{*} f\right)=f$. We may therefore assume that $e A e$ is a division algebra and consequently that $\operatorname{rank} e=1$. Since $\left(1-e^{*}\right)(1-e)=1-\left(e+e^{*}-e^{*} e\right)=0$, a repetition of the above argu- 
ment allows us to assume that $1-e$ is also an idempotent of rank 1 . It follows from Remark 1 that $A$ is the complete ring of linear transformations of a two dimensional vector space $V$ over a division algebra $\Delta$.

If $e^{*} e=0$ as well as $e e^{*}=0$ it is easy to show that relative to a suitable basis of $V e=E_{11}$ and $e^{*}=E_{22}$. In this case we are finished. Therefore suppose $e^{*} e \neq 0$. We shall sketch an argument, leaving some details to the reader, whereby a non-trivial symmetric idempotent can now be found. First find a basis $\left(u_{1}, u_{2}\right)$ of $V$ such that $u_{1} e=u_{1}, u_{2} e=0$, $u_{1} e^{*}=0, u_{2} e^{*}=\lambda u_{1}+u_{2}$, where $\lambda \neq 0 \in \Delta$. By setting $v_{1}=\lambda^{-1} u_{1}$ and $v_{2}=u_{2}$ we obtain a basis $\left(v_{1}, v_{2}\right)$ of $V$ relative to which $e=E_{11}$ and $e^{*}=E_{21}+E_{22}$. From this we have

$$
\begin{aligned}
& E_{11}^{*}=E_{21}+E_{22} \\
& E_{21}^{*}=\left[\left(E_{21}+E_{22}\right) E_{11}\right]^{*}=\left(E_{21}+E_{22}\right) E_{11}=E_{21} \\
& E_{22}^{*}=e-E_{21}^{*}=E_{11}-E_{21} .
\end{aligned}
$$

Set $E_{12}^{*}=\alpha E_{11}+\beta E_{12}+\gamma E_{21}+\delta E_{22}, \alpha, \beta, \gamma, \delta \in \Delta$. From the following three equations

$$
\begin{aligned}
& E_{11}-E_{21}=E_{22}^{*}=\left(E_{21} E_{12}\right)^{*}=E_{12}^{*} E_{21}^{*}=\beta E_{11}+\delta E_{21} \\
& E_{21}+E_{22}=E_{11}^{*}=\left(E_{12} E_{21}\right)^{*}=E_{21}^{*} E_{12}^{*}=\alpha E_{21}+\beta E_{22} \\
& \begin{aligned}
\alpha E_{11}+\beta E_{12}+\gamma E_{21}+\delta E_{22}=E_{12}^{*} & =\left(E_{11} E_{12}\right)^{*}=E_{12}^{*} E_{11}^{*} \\
& =\beta E_{11}+\beta E_{12}+\delta E_{21}+\delta E_{22}
\end{aligned}
\end{aligned}
$$

we obtain $\alpha=1, \beta=1, \gamma=-1$, and $\delta=-1$. Hence

$$
E_{12}^{*}=E_{11}+E_{12}-E_{21}-E_{22}
$$

and $-E_{12} E_{12}^{*}=E_{11}+E_{12}$ is then a non-trivial symmetric idempotent.

There remains the case in which $x x^{*} \neq 0$ for all $x \neq 0 \in A$. We note that in this situation there exist no nonzero nilpotent symmetric elements, for, if $s \neq 0$ is symmetric, then $s^{2}=s s^{*} \neq 0$. If $A$ is not already a division algebra then we can find an element $x \neq 0 \in A$ such that $x A$ is a proper right ideal. It follows that $x x^{*} A \subseteq x A$ is also a proper right ideal, and so $x x^{*}$ is a nonzero, and hence, non-nilpotent symmetric element which has no inverse. In case $A$ is algebraic over $\Phi$ the subalgebra $\left[\left[x x^{*}\right]\right]$ generated by $x x^{*}$ contains a non-trivial symmetric idempotent, by Remark 4.

\section{Total matrix rings with involution. We begin by proving}

THEOREM 4. Let $A$ be the total matrix ring $\Delta_{m}$ with an involution *, where $\triangle$ is a division algebra over $\Phi$. Then there exists a set of orthogonal symmetric elements $e_{1}, e_{2}, \cdots, e_{m_{1}}, f_{1} f_{2}, \cdots, f_{m_{2}}$ such that:

(a) The $e_{i}$ are non-nilpotent elements of rank 1 . In case $A$ is 
algebraic over $\Phi$, the $e_{i}$ are idempotents of rank 1.

(b) The $f_{j}$ are idempotents of rank 2 , and $f_{j} A f_{j}$ is isomorphic to $\Delta_{2}$, with $E_{11}^{*}=E_{22}$ (see Theorem 3).

(c) $m_{1}+2 m_{2}=m$.

Proof. Let $s_{1}, s_{2}, \cdots, s_{h}$ be a set of nonzero orthogonal symmetric idempotents, with $h$ maximal. By the maximality of $h$ we have

$$
s_{1}+s_{2}+\cdots+s_{h}=1 .
$$

Each $s_{i} A s_{i}$ may itself be regarded as a total matrix ring $\Delta_{r_{i}}$ with an involution induced by ${ }^{*}$, where $r_{i}$ is the rank of $s_{i}$. We first consider those $s_{i} A s_{i}$ having the property: there exists an $x \neq 0 \in s_{i} A s_{i}$ such that $x x^{*}=0$. Theorem 3 , together with the maximality of $h$, then says that $s_{i} A s_{i}$ is isomorphic to $\Delta_{2}$, with $E_{11}^{*}=E_{22}$. Relabeling these $s_{i}$ as $f_{1}, f_{2}, \cdots, f_{m_{2}}$, we have taken care of $(b)$.

The remaining $s_{i}$, of course, have the property that $x x^{*} \neq 0$ for all $x \neq 0 \in s_{i} A s_{i}$. As we have noted before, $s_{i} A s_{i}$ can have no nonzero nilpotent symmetric elements, since $x x^{*} \neq 0$. Consider a typical $s_{i} A s_{i}$ and select from it an element $x_{1}$ of rank 1 . Then $y_{1}=x_{1} x_{1}^{*} \neq 0$ is a non-nilpotent symmetric element of rank 1. Now assume that $k\left(<r_{i}\right)$ orthogonal non-nilpotent symmetric elements $y_{1}, y_{2}, \cdots, y_{k}$ of rank 1 have been found. Since the dimension of $W=\sum_{l=1}^{k} V y_{i}$ is less than $r_{i}$, we can find an element $x_{k+1}$ of rank 1 such that $W x_{k+1}=0$. Then $y_{k+1}=$ $x_{k+1} x_{k+1}^{*}$ is a non-nilpotent symmetric element of rank 1 such that $W y_{k+1}=0$, that is, $y_{i} y_{k+1}=0, i=1,2, \cdots, k$. Also $y_{k+1} y_{i}=0, i=$ $1,2, \cdots, k$, since $\left(y_{k+1} y_{i}\right)^{*}=y_{i}^{*} y_{r+1}^{*}=y_{i} y_{k+1}=0$. It follows that there exists in $s_{i} A s_{i}$ a set of $r_{i}$ non-nilpotent orthogonal symmetric elements $y_{1}, y_{2}, \cdots, y_{r_{i}}$, each of rank 1 . If $A$ is algebraic over $\Phi$ the subalgebra [ $\left.\left[y_{j}\right]\right]$ generated by each $y_{j}$ contains a nonzero idempotent $z_{j}$ (necessarily of rank 1), and so we have $r_{i}$ orthogonal symmetric idempotents $z_{1}, z_{2}, \cdots, z_{r_{i}}$, each of rank 1 . Repeating the argument for all the $s_{i} A s_{i}$ and labeling either all the $y_{j}$ or all the $z_{j}$ as $e_{1}, e_{2}, \cdots, e_{m_{1}}$, we have completed the proof of (a). (c) follows readily from the fact that $\operatorname{rank} e_{i}=1, \operatorname{rank} f_{j}=2$, and $\sum_{i} e_{i}+\sum_{j} f_{j}=1$.

To illustrate Theorem 4 we consider the following simple example. Let $A=\Phi_{2}$, where $\Phi$ is a field, and define an involution * in $A$ by:

$$
\left(\begin{array}{ll}
\alpha_{1} & \alpha_{2} \\
\alpha_{3} & \alpha_{4}
\end{array}\right)^{*}=\left(\begin{array}{cc}
0 & -1 \\
1 & 0
\end{array}\right)\left(\begin{array}{cc}
\alpha_{1} & \alpha_{3} \\
\alpha_{2} & \alpha_{4}
\end{array}\right)\left(\begin{array}{cc}
0 & 1 \\
-1 & 0
\end{array}\right), \alpha_{i} \in \Phi
$$

The reader may verify that $A$ contains no symmetric elements of rank 1. Similar examples of higher dimension can also be given.

In the remainder of this section we derive a result which will enable us, at least in the algebraic case, to "pass" from the total matrix ring 
$\Delta_{m}$ to the division algebra $\Delta$ itself.

LEMMA 2. Let $A$ be the total matrix ring $\Delta_{2}$, algebraic over $\Phi$, with an involution *, where $\Delta$ is a division algebra over $\Phi$. Suppose $E_{11}^{*}=E_{22}$. Then one of the following two possibilities must hold:

(a) A contains a symmetric idempotent of rank 1 .

(b) The involution * in $A_{2}$ is of the form:

$$
\left(\begin{array}{ll}
\alpha_{1} & \alpha_{2} \\
\alpha_{3} & \alpha_{4}
\end{array}\right)^{*}=\left(\begin{array}{cc}
0 & -\beta^{-1} \\
\beta^{-1} & 0
\end{array}\right)\left(\begin{array}{ll}
\bar{\alpha}_{1} & \bar{\alpha}_{3} \\
\bar{\alpha}_{2} & \bar{\alpha}_{4}
\end{array}\right)\left(\begin{array}{cc}
0 & \beta \\
-\beta & 0
\end{array}\right)
$$

for all $\alpha_{\imath} \in \Delta$, some $\beta \neq 0 \in \Delta$, where $\alpha \rightarrow \bar{\alpha}$ is an involution in $\Delta$.

Proof. It is well known (see for example [2], p. 24, Theorem 9) that the involution * in $A$ has the form:

$$
\left(\begin{array}{ll}
\alpha_{1} & \alpha_{2} \\
\alpha_{3} & \alpha_{4}
\end{array}\right)^{*}=U^{-1}\left(\begin{array}{ll}
\bar{\alpha}_{1} & \bar{\alpha}_{3} \\
\bar{\alpha}_{2} & \bar{\alpha}_{4}
\end{array}\right) U
$$

where $U=\left(\begin{array}{cc}\gamma & \beta \\ \pm \bar{\beta} & \delta\end{array}\right)$ is a nonsingular element of $\Delta_{2}$ and $\alpha \rightarrow \bar{\alpha}$ is an involution in 4 . Consider the equation $E_{22}=E_{11}^{*}=U^{-1} E_{11} U$, that is,

$$
\left(\begin{array}{cc}
\gamma & \beta \\
\pm \bar{\beta} & \delta
\end{array}\right)\left(\begin{array}{ll}
0 & 0 \\
0 & 1
\end{array}\right)=\left(\begin{array}{ll}
1 & 0 \\
0 & 0
\end{array}\right)\left(\begin{array}{cc}
\gamma & \beta \\
\pm \bar{\beta} & \delta
\end{array}\right)
$$

It follows that $\gamma=\delta=0$, and hence $U=\left(\begin{array}{cc}0 & \beta \\ \pm \bar{\beta} & 0\end{array}\right)$.

At this point we observe that an element $\left(\begin{array}{ll}\gamma_{1} & \gamma_{2} \\ \gamma_{1} & \gamma_{2}\end{array}\right) \in A$ is a nonnilpotent element of rank 1 , unless $\gamma_{1}+\gamma_{2}=0$. Now set $B=\left(\begin{array}{cc} \pm \bar{\beta} & \beta \\ \pm \bar{\beta} & \beta\end{array}\right)$. It is easy to check that $B^{*}=U^{-1}\left(\begin{array}{cc} \pm \beta & \pm \beta \\ \bar{\beta} & \bar{\beta}\end{array}\right) U= \pm B$, and hence $B$ is either symmetric or skew. If $\beta \pm \bar{\beta}=0$, i.e., $U=\left(\begin{array}{cc}0 & \beta \\ -\beta & 0\end{array}\right)$, we are finished. Therefore assume that $\beta \pm \bar{\beta} \neq 0$. We then apply the observation made at the beginning of this paragraph to conclude that $B$ is a non-nilpotent element of rank 1 . Since $B$ is either symmetric or skew, it follows that $B^{2}$ is a non-nilpotent symmetric element of rank 1. The proof is complete when we note that, as $A$ is algebraic over $\Phi$, the subalgebra $\left[\left[B^{2}\right]\right]$ generated by $B^{2}$ over $\Phi$ contains a symmetric idempotent of rank 1 .

THeOREM 5. Let $A$ be the total matrix ring $\Delta_{m}$, algebraic over $\Phi$, with an involution *, where $\Delta$ is a division algebra over $\Phi$. Then there exists a division subalgebra $D$ of $A$ such that $D^{*}=D$ and $D$ is isomorphic to 4 . 
Proof. Theorem 4 asserts the existence of either (a) a symmetric idempotent $e$ of rank 1 or (b) a symmetric idempotent $f$ of rank 2, where $f A f$ is isomorphic to $A_{2}$ with the induced involution * such that $E_{11}^{*}=E_{22}$. In case (a) we merely set $D=e A e$ and the required conclusion follows. In case (b) $\Delta_{2}$ satisfies the hypothesis of Lemma 2. If $\Delta_{2}$ contains a symmetric idempotent of rank 1 we proceed as in case (a). Otherwise we conclude from Lemma 2 that the involution $*$ in $\Delta_{2}$ is given by:

$$
\left(\begin{array}{ll}
\alpha_{1} & \alpha_{2} \\
\alpha_{3} & \alpha_{4}
\end{array}\right)^{*}=\left(\begin{array}{cc}
0 & -\beta^{-1} \\
-\beta^{-1} & 0
\end{array}\right)\left(\begin{array}{ll}
\bar{\alpha}_{1} & \bar{\alpha}_{3} \\
\bar{\alpha}_{2} & \bar{\alpha}_{4}
\end{array}\right)\left(\begin{array}{cc}
0 & \beta \\
-\beta & 0
\end{array}\right)
$$

Let $D$ be the division subalgebra of $A_{2}$ consisting of all elements of the form $\left\{\begin{array}{ll}\alpha & 0 \\ 0 & \alpha\end{array}\right\}, \alpha \in \Delta . \quad D$ is obviously isomorphic to $\Delta$. Furthermore, one verifies that

$$
\left\{\begin{array}{ll}
\alpha & 0 \\
0 & \alpha
\end{array}\right\}^{*}=\left\{\begin{array}{cc}
\beta^{-1} \bar{\alpha} \beta & 0 \\
0 & \beta^{-1} \bar{\alpha} \beta
\end{array}\right\} \in D
$$

and we see that $D^{*}=D$.

5. Division $S_{n}$-algebras. We begin this section by stating

LEMma 3. Let $\triangle$ be an algebraic division algebra over its center $\Phi$ for which there exists a fixed integer $h$ such that the dimension of $\Phi(x)$ over $\Phi$ is equal to or less than $h$ for every separable element $x \in \Delta$. Then $\Delta$ is finite dimensional over $\Phi$.

Except for the restriction of separability, this lemma is virtually the same as [1], p. 181, Theorem 1. The proof appearing in [1] carries over directly, and we therefore omit it.

Lemma 4. Let $\triangle$ be an algebraic $S_{n}$-division algebra of the first kind over its center $\Phi$. Suppose $E$ is a finite dimensional field extension of $\Phi$. Then $E \otimes_{\phi} \Delta$ is isomorphic to the total matrix ring $\Gamma_{m}$, where $\Gamma$ is a division algebra and $m \leqq 2 n$.

Proof. $E \otimes \Delta$ is well known to be a simple algebra over $\Phi$ with minimum condition on right ideals. Hence $E \otimes \Delta$ is isomorphic to $\Gamma_{m}$, where $\Gamma$ is a division algebra and $m$ is a natural number.

An involution $\tau$ can be defined in $E \otimes \Delta$ as follows:

$$
(\alpha \otimes x)^{\tau}=\alpha \otimes x^{*}
$$

for $\alpha \in E, x \in \Delta$. It can be verified that $\tau$ is a well-defined involution 
and that every symmetric element (under $\tau$ ) in $E \otimes \Delta$ can be written in the form:

$$
\sum_{i} \alpha_{i} \otimes s_{i}, \alpha_{i} \in E, s_{i} \in S .
$$

Let $f\left(t_{1}, t_{2}, \cdots, t_{n}\right)=0$ be the multilinear polynomial identity of degree $n$ satisfied by $S$. Because this identity is multilinear and because $E$ is the center of $E \otimes \Delta$, it follows from (3) that the set of symmetric elements of $E \otimes \Delta$ under $\tau$ also satisfies $f\left(t_{1}, t_{2}, \cdots, t_{n}\right)=0$.

Now regard $E \otimes \Delta$ as the total matrix ring $\Gamma_{m}$, with involution $\tau$. By Theorem 4 there exists in $\Gamma_{m}$ a set of at least $k$ non-nilpotent orthogonal symmetric elements, where $2 k \geqq m$. Theorem 2 tells us that $k \leqq n$, and hence $m \leqq 2 k \leqq 2 n$.

We are now able to prove

THEOREm 6. Let $\Delta$ be an algebraic $S_{n}$-division algebra. Then $\Delta$ is finite dimensional over its center.

Proof. By Theorem 1 we may assume that $\Delta$ is an $S_{n}$-algebra of the first kind over its center $\Phi$. Suppose $\Delta$ is not finite dimensional over $\Phi$. Then by Lemma 3 there exists a separable element $x \in \Delta$ whose minimal polynomial $g(t)$ over $\Phi$ has degree $r>2 n$. Let $E$ be a finite dimensional field extension of $\Phi$ containing the $r$ distinct roots $\alpha_{1}, \alpha_{2}, \cdots, \alpha_{r}$ of $g(t)$.

We claim now that the element $x-\alpha_{i}$ is a zero divisor in $E \otimes \Delta$, $i=1,2, \cdots, r$. Indeed,

$$
0=g(x)=\prod_{j=1}^{r}\left(x-\alpha_{j}\right)=\left(x-\alpha_{i}\right) \prod_{j \neq i}\left(x-\alpha_{j}\right),
$$

and it suffices to show that $\Pi_{j \neq i}\left(x-\alpha_{j}\right)$ is a nonzero element of $E \otimes \Delta$. Suppose $\Pi_{j \neq i}\left(x-\alpha_{j}\right)=0$, that is,

$$
\left(x^{r-1} \otimes 1\right)-\left(x^{r-2} \otimes \sum_{j \neq i} \alpha_{j}\right)+\cdots \pm\left(1 \otimes \prod_{j \neq i} \alpha_{j}\right)=0 .
$$

Since $x^{r-1}, x^{r-2}, \cdots, 1$ are linearly independent over $\Phi$, all the corresponding terms of $E$ in (4) must be zero, which is clearly impossible. Therefore $x-\alpha_{i}$ is a zero divisor in $E \otimes \Delta$.

According to Lemma $4 E \otimes \Delta$ is isomorphic to the total matrix ring $\Gamma_{m}$, where $m \leqq 2 n$. We may therefore regard $E \otimes \Delta$ as the complete ring of linear transformations of an $m$-dimensional vector space $V$ over the division algebra $\Gamma$. Set $V_{i}=\left\{v \in V \mid v\left(x-\alpha_{i}\right)=0\right\}, i=1,2, \cdots, r$. $V_{i}$ is a nonzero subspace of $V$ since $x-\alpha_{i}$ is a zero divisor in $E \otimes \Delta$. Using the fact that the $\alpha_{i}$ are distinct elements belonging to the center $E$, we have that $V_{i}$ are independent subspaces of $V$. It follows that 


$$
m \geqq \operatorname{dim} \sum_{i=1}^{r} V_{i}=\sum_{i=1}^{r}\left(\operatorname{dim} V_{i}\right) \geqq r>2 n .
$$

A contradiction now arises since $m \leqq 2 n$. We must therefore conclude that $\Delta$ is finite dimensional over its center.

6. Primitive $S_{n}$-algebras. We are now in a position to proceed with the proof of our main result.

TheOREM 7. Let $A$ be a primitive algebraic $S_{n}$-algebra. Then the center of $A$ is a field, and $A$ is finite dimensional over its center.

Proof. Since $A$ is primitive, $A$ may be regarded as a dense ring of linear transformations of a vector space $V$ over a division algebra 4. According to Theorem 2 there exist at most $n$ orthogonal symmetric idempotents. Let $e_{1}, e_{2}, \cdots, e_{m}$ be a set of $m$ orthogonal symmetric idempotents, with $m(\leqq n)$ maximal. For each $i, e_{i} A e_{i}$ is again a primitive algebraic algebra with involution induced by *. The same is true for $(1-e) A(1-e)$, where $e=e_{1}+e_{2}+\cdots+e_{m}$, if $A$ should not already happen to have an identity. We now use Theorem 3 in conjunction with the maximality of $m$ to assert that the rank of each $e_{i}$ is 1 or 2 , and that $A$ does have an identity $1=e_{1}+e_{2}+\cdots+e_{m}$. It follows that the dimension $k$ of $V \leqq 2 m$ and consequently that $A$ is isomorphic to the total matrix ring $\Delta_{k}$. The center of $A$ is, of course, a subfield of $\Delta$. Theorem 5 now says that $\Delta$ is an algebraic $S_{n}$-division algebra. By Theorem $6 \Delta$ is finite dimensional over its center. Hence $A$ is finite dimensional over its center.

COROLlaRY. Let $A$ be a primitive algebraic algebra with an involution * such that the set $K$ of skew elements is a PI-subspace of degree $n$. Then $A$ is finite dimensional over its center.

Proof. Let $f\left(t_{1}, t_{2}, \cdots, t_{n}\right)=0$ be the multilinear polynomial identity of degree $n$ satisfied by $K$, according to Lemma 1 . If $s_{1}, s_{2} \in S$, where $S$ is the set of symmetric elements of $A$, then $s_{1} s_{2}-s_{2} s_{1} \in K$. From this it follows that $f\left(u_{1} v_{1}-v_{1} u_{1}, u_{2} v_{2}-v_{2} u_{2}, \cdots, u_{n} v_{n}-v_{n} u_{n}\right)=0$ is a nontrivial polynomial identity of degree $2 n$ satisfied by the elements of $S$. In other words, $A$ is a primitive algebraic $S_{2 n}$-algebra, and the conclusion follows from Theorem 7 .

Note. Herstein's original conjecture was: if $A$ is a simple ring (or algebra) with involution whose skew elements satisfy a polynomial identity, then $A$ is finite dimensional over its center. In this paper we have verified his conjecture in the special case where $A$ is a simple algebraic algebra which is not a nil algebra. 


\section{BibLIOGRAPHY}

1. N. Jacobson, Structure of rings, Amer. Math. Soc. Colloquium Publications, 37 (1956).

2. The theory of rings, Amer. Math. Soc. Math. Surveys, vol. 2, New York 1943.

UNIVERSITY OF CHICAGO

SMITH COLLEGE 



\section{PACIFIC JOURNAL OF MATHEMATICS}

\section{EDITORS}

\author{
RaLPh S. Phillips \\ Stanford University \\ Stanford, California \\ F. H. BRowNELL \\ University of Washington \\ Seattle 5 , Washington
}

A. L. Whiteman

University of Southern California

Los Angeles 7, California

L. J. Paige

University of California

Los Angeles 24, California

\author{
E. F. BECKENBACH \\ T. M. CHERRY
}

\author{
ASSOCIATE EDITORS

$\begin{array}{lll}\text { D. DERRY } & \text { H. L. ROYDEN } & \text { E. G. STRAUS } \\ \text { M. OHTSUKA } & \text { E. SPANIER } & \text { F. WOLF }\end{array}$

\section{SUPPORTING INSTITUTIONS}

\author{
UNIVERSITY OF BRITISH COLUMBIA \\ CALIFORNIA INSTITUTE OF TECHNOLOGY \\ UNIVERSITY OF CALIFORNIA \\ MONTANA STATE UNIVERSITY \\ UNIVERSITY OF NEVADA \\ NEW MEXICO STATE UNIVERSITY \\ OREGON STATE COLLEGE \\ UNIVERSITY OF OREGON \\ OSAKA UNIVERSITY \\ UNIVERSITY OF SOUTHERN CALIFORNIA
}

\author{
STANFORD UNIVERSITY \\ UNIVERSITY OF TOKYO \\ UNIVERSITY OF UTAH \\ WASHINGTON STATE COLLEGE \\ UNIVERSITY OF WASHINGTON \\ AMERICAN MATHEMATICAL SOCIETY \\ CALIFORNIA RESEARCH CORPORATION \\ HUGHES AIRCRAFT COMPANY \\ SPACE TECHNOLOGY LABORATORIES \\ NAVAL ORDNANCE TEST STATION
}

Mathematical papers intended for publication in the Pacific Journal of Mathematics should be typewritten (double spaced), and the author should keep a complete copy. Manuscripts may be sent to any one of the four editors. All other communications to the editors should be addressed to the managing editor, L. J. Paige at the University of California, Los Angeles 24, California.

50 reprints per author of each article are furnished free of charge; additional copies may be obtained at cost in multiples of 50 .

The Pacific Journal of Mathematics is published quarterly, in March, June, September, and December. The price per volume (4 numbers) is $\$ 12.00$; single issues, $\$ 3.50$. Back numbers are available. Special price to individual faculty members of supporting institutions and to individual members of the American Mathematical Society: $\$ 4.00$ per volume; single issues, $\$ 1.25$.

Subscriptions, orders for back numbers, and changes of address should be sent to Pacific Journal of Mathematics, 103 Highland Boulevard, Berkeley 8, California.

Printed at Kokusai Bunken Insatsusha (International Academic Printing Co., Ltd.), No. 6, 2-chome, Fujimi-cho, Chiyoda-ku, Tokyo, Japan.

\section{PUBLISHED BY PACIFIC JOURNAL OF MATHEMATICS, A NON-PROFIT CORPORATION}

The Supporting Institutions listed above contribute to the cost of publication of this Journal, but they are not owners or publishers and have no responsibility for its content or policies.

Reprinted 1966 in the United States of America 


\section{Pacific Journal of Mathematics}

\section{Vol. 11, No. 4}

A. V. Balakrishnan, Prediction theory for Markoff processes . . . . . . . . . . 1171

Dallas O. Banks, Upper bounds for the eigenvalues of some vibrating systems . . . . 1183

A. Białynicki-Birula, On the field of rational functions of algebraic groups ...... 1205

Thomas Andrew Brown, Simple paths on convex polyhedra .............. 1211

L. Carlitz, Some congruences for the Bell polynomials . . . . . . . . . . . . 1215

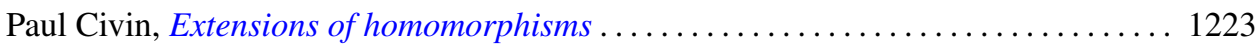

Paul Joseph Cohen and Milton Lees, Asymptotic decay of solutions of differential

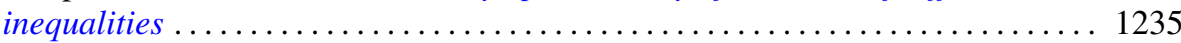

István Fáry, Self-intersection of a sphere on a complex quadric . . . . . . . . . . 1251

Walter Feit and John Griggs Thompson, Groups which have a faithful representation

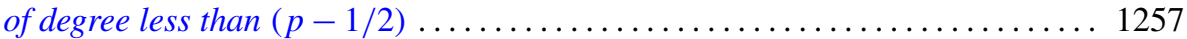

William James Firey, Mean cross-section measures of harmonic means of convex

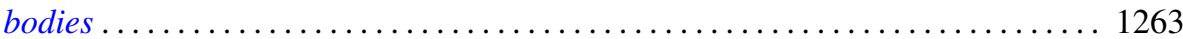

Avner Friedman, The wave equation for differential forms . . . . . . . . . . 1267

Bernard Russel Gelbaum and Jesus Gil De Lamadrid, Bases of tensor products of

Banach spaces ................................... 1281

Ronald Kay Getoor, Infinitely divisible probabilities on the hyperbolic plane . . . . 1287

Basil Gordon, Sequences in groups with distinct partial products . . . . . . . . . . . . 1309

Magnus R. Hestenes, Relative self-adjoint operators in Hilbert space . . . . . . . . . 1315

Fu Cheng Hsiang, On a theorem of Fejér ......................... 1359

John McCormick Irwin and Elbert A. Walker, On N-high subgroups of Abelian



John McCormick Irwin, High subgroups of Abelian torsion groups . . . . . . . . . 1375

R. E. Johnson, Quotient rings of rings with zero singular ideal . . . . . . . . . . . 1385

David G. Kendall and John Leonard Mott, The asymptotic distribution of the time-to-escape for comets strongly bound to the solar system ...

Kurt Kreith, The spectrum of singular self-adjoint elliptic operators ....

Lionello Lombardi, The semicontinuity of the most general integral of the calculus of variations in non-parametric form ................................

Albert W. Marshall and Ingram Olkin, Game theoretic proof that Chebyshev inequalities are sharp

Wallace Smith Martindale, III, Primitive algebras with involution . . William H. Mills, Decomposition of holomorphs ..............

James Donald Monk, On the representation theory for cylindric algebras . . . . . . 1447

Shu-Teh Chen Moy, A note on generalizations of Shannon-McMillan theorem . . . . 1459

Donald Earl Myers, An imbedding space for Schwartz distributions . .

John R. Myhill, Category methods in recursion theory .........

Paul Adrian Nickel, On extremal properties for annular radial and circular slit mappings of bordered Riemann surfaces

Edward Scott O'Keefe, Primal clusters of two-element algebras . .

Nelson Onuchic, Applications of the topological method of Wazewski to certain

problems of asymptotic behavior in ordinary differential equations ...

Peter Perkins, A theorem on regular matrices................

Clinton M. Petty, Centroid surfaces .... 